\begin{tabular}{|c|l|}
\hline Title & $\begin{array}{l}\text { Formation of High-Density GaA s Hexagonal Nano-wire Networks by Selective MBE Growth on Pre patterned (001) } \\
\text { Substrates }\end{array}$ \\
\hline Author(s) & Tamai, Isao; Sato, Taketomo; Hasegawa, Hideki \\
\hline Citation & $\begin{array}{l}\text { Physica E: Low-dimensional Systems and Nanostructures, 21(2-4), 521-526 } \\
\text { https://doi.org/10.1016/.physe.2003.11.080 }\end{array}$ \\
\hline Issue Date & 200403 \\
\hline Doc URL & http://hdl.handle.net/2115/8387 \\
\hline Type & article (author version) \\
\hline File Information & TSA TO49.PDF \\
\hline
\end{tabular}

Instructions for use 


\title{
Formation of High-Density GaAs Hexagonal Nano-wire Networks by Selective MBE Growth on Pre-patterned (001) Substrates
}

\author{
Isao Tamai, Taketomo Sato and Hideki Hasegawa \\ Research Center for Integrated Quantum Electronics and Graduate School of Electronics and Information Engineering, \\ Hokkaido University, N-13, W-8, Kita-Ku, Sapporo 060-8628, Japan \\ Phone: +81-11-706-7172, Fax+81-11-716-6004, Email: tamai@rciqe.hokudai.ac.jp
}

\begin{abstract}
Attempts were made to grow high-density GaAs hexagonal nano-wire networks on (001) patterned substrates by selective molecular beam epitaxy (MBE). To form a hexagon, $\langle\overline{1} 10>$ - and $<510>$ - directions were combined. By the growth of straight wire arrays in each direction, the growth mode, conditions and mechanism were investigated. The wire width was shown to be determined for both directions by the facet boundary planes resulting from the growth rate difference on different facets. By optimizing of growth condition, highly uniform and smoothly connected hexagonal nano-wire networks with a density of $3 \times 10^{8} \mathrm{~cm}^{-2}$ were successfully formed.
\end{abstract}

Keywords: selective MBE growth, quantum wire, network, GaAs, AlGaAs

\section{Introduction}

Recently, intensive research efforts have been made on semiconductor quantum devices such as single electron transistors and quantum wire transistors. For realization of high-density and large-scale integration of quantum nano-devices, it is necessary to form high quality and high-density network of quantum structures in a size- and position-controlled fashion.

Among various approaches to form quantum structures, selective molecular beam epitaxy (MBE) and metalorganic vapor phase epitaxy (MOVPE) growth on pre-patterned substrates is one of the most promising techniques for formation of position- and size-controlled arrays of III-V quantum wires (QWRs) and quantum dots (QDs) [1-7].

The purpose of this paper is to attempt to realize high-density GaAs hexagonal nano-wire networks by selective MBE growth on pre-patterned (001) substrates by the proper understanding of the growth modes, conditions and mechanism. Such hexagonal nano-wire networks are promising structures to implement hexagonal binary decision diagram (BDD) quantum circuits recently proposed by our group for quantum LSIs $[8,9]$.

\section{Experimental}

Figures 1 (a)-(c) show substrate patterns used for selective growth experiments. To form a hexagon, two kinds of wire direction are required. In this study, $<\overline{1} 10>$ - and $<510>$-orientations were investigated as candidates of wire directions in Figs.1 (a) and (b). This combinations produce hexagonal patterns close to the hexagonal close packed structure with an interior angle of about $120^{\circ}$ as shown in Fig.1 (c). Attempts to grow straight QWRs were made first, using straight striped patterns shown in Figs.1 (a) and (b). Then, after optimization of growth process for each wire directions, hexagonal nano-wire networks were grown on the patterned substrates shown in Fig.1 (c). Substrate patterns were prepared by a standard electron-beam lithography and $\mathrm{H}_{2} \mathrm{SO}_{4}$ - or $\mathrm{HBr}$ - based wet chemical etching.

Typical material supply sequence used in the experiment is shown in Fig.1 (d) in terms of growth on a planar substrate which was placed next to the non-planar substrate. The growth sequence is similar to that for growth of InP-based ridge QWRs [4]. Namely, after a standard thermal cleaning in the MBE chamber for native oxide removal, GaAs ridge structures was grown first. Then, attempts of self-organized QWR growth were made by supplying $\mathrm{Al}_{0.3} \mathrm{Ga}_{0.7} \mathrm{As} / \mathrm{GaAs} / \mathrm{Al}_{0.3} \mathrm{Ga}_{0.7} \mathrm{As}$ sandwiched layers on the ridge structure. The supply rate of the AlGaAs layer was kept to be $500 \mathrm{~nm} /$ hour at a V/III flux ratio of 30 in terms of the value for growth on a planar (001) surface. Growth temperature was $640^{\circ} \mathrm{C}$.

\section{Selective Growth of Straight Nano-wires}

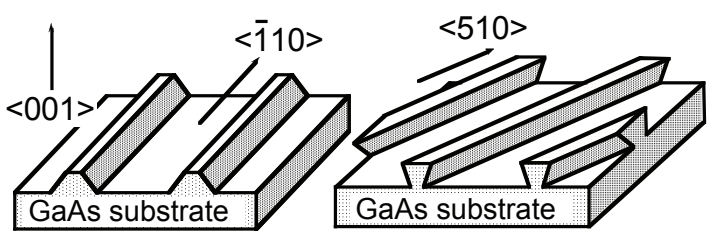

(a)

(b)

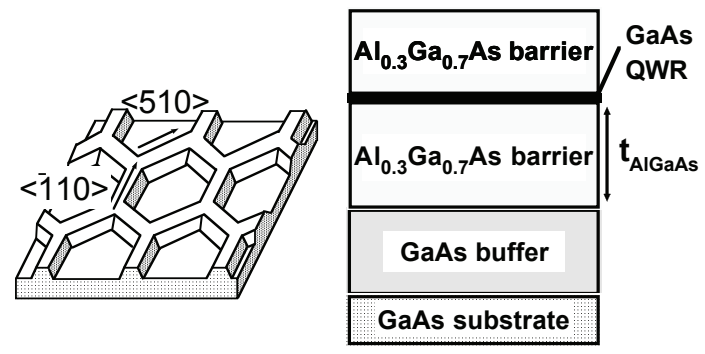

(c)

(d)

Fig.1 Substrate patterns (a)-(c); (a) for $<\overline{1} 10>$-wires (b) for $<510>$-wire and (c) for hexagonal networks. (d) Growth on a planar substrate. 
3.1 Cross-sectional features of $<\overline{1} 10>-$ and $<510>$ oriented nano-wires

Figures 2 (a) and (b) show cross-sectional SEM images of $<\overline{1} 10>$ - and $<510>$ - oriented QWRs grown at $640^{\circ} \mathrm{C}$, respectively. In the case of mesa stripes along the $<\overline{1} 10>$-direction, growth of GaAs buffer layer led to formation of GaAs ridge structures defined by two (113) facets. Then, supply of AlGaAs/GaAs/AlGaAs materials resulted in self-organized formation of arrow-head shaped nano-wire on the top (113) facets of the GaAs ridge structure as shown in Fig.2 (a). Furthermore, two facet boundary planes separating (111)/(113) growth region became also visible within the AlGaAs layer after stain etching by alkali solution where GaAs dissolves selectively into the solution. Thus, the wire width, w, is determined by the separation of two (113)/(111) facet boundaries just prior to GaAs supply for QWR growth.

In the case of the $<510>$-direction, wet chemical etching produced the inverse-mesa stripes on substrates. Growth of GaAs ridge on inverse-mesa stripes led to formation of flat-top ridge structures defines by top (001) plane and (155) side facets. As shown in Fig.2 (b), subsequent AlGaAs/GaAs/AlGaAs supply resulted in self-organized formation of QWRs on the top plane with decreased sizes. Although not visible by stain etching, repeated growth experiments indicated that the wire width reduced again by following the facet boundary planes in the AlGaAs layer, as shown in Fig.3.

\subsection{Growth mode and mechanism}

The width of the ridge nano-wire was investigated vs. the growth time of the lower AlGaAs layer both for
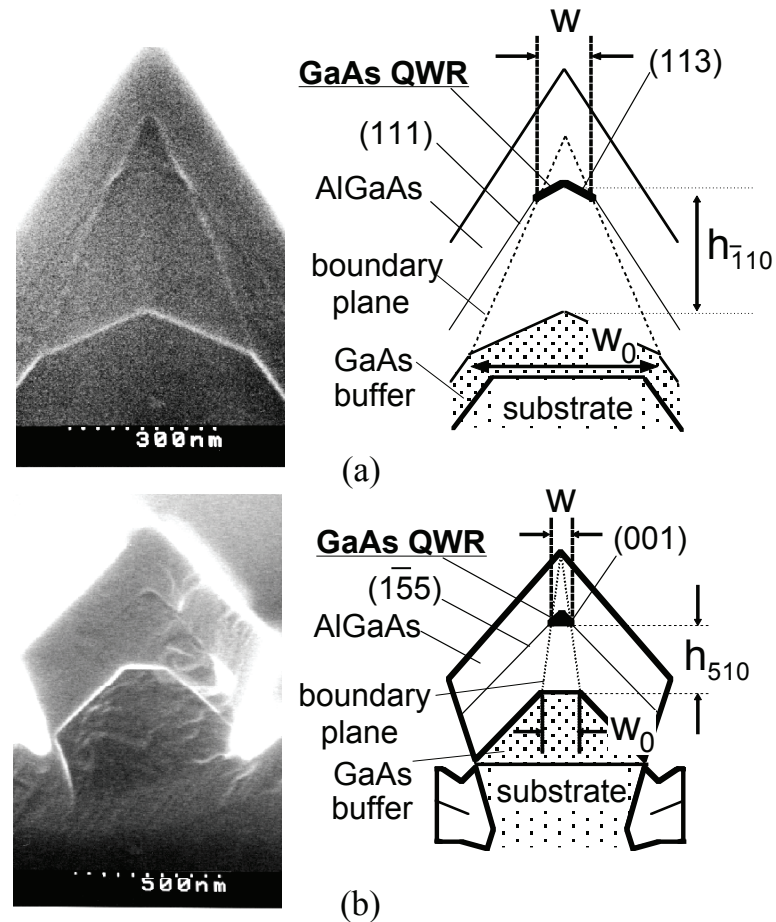

Fig.2 Cross-sectional SEM images of (a) $<\overline{1} 10>$ oriented QWRs and (b) $<510>$-oriented QWRs $<\overline{1} 10>$ - and $<510>$-directions. The observed values of the ratio of the wire width, $w$, to the initial lateral GaAs ridge width, $\mathrm{w}_{0}$, prior to $\mathrm{AlGaAs}$ growth are plotted in Fig.4 as a function of the AlGaAs supply thickness, $t_{\mathrm{AlGaAs}}$. As seen in Fig.4, the wire width, w, changed linearly with the value of $t_{\mathrm{AlGaAs}}$, both for $\langle\overline{1} 10>$ - and $<510>$-oriented wires. However, the sensitivity slope was different for two wire directions.

As for the growth mechanism causing such linear reduction, our previous detailed study [7] on the growth of the $\langle\overline{1} 10>$-oriented wire has shown that it is due to a larger growth rate of AlGaAs on the (113) facet than on the (111) facet. Furthermore, the difference in migration and atom incorporation rates leads to a Ga-rich thicker region on (113) facet, and a less Ga-rich thinner region on (111) facet. Due to composition difference, the boundary becomes visible by stain etching for cross-sectional SEM observations shown in Fig.2 (a).

A similar experiment on the $<510>$-oriented ridge has shown that the growth rate of AlGaAs on the (001) facet of ridge top is larger than that on the side $(1 \overline{5} 5)$ facets. This explains the observed reduction of the wire width. The reason why the boundary plane is not clearly seen in the cross-section of $<510>$-oriented wire is most probably due to smaller difference of Al incorporation

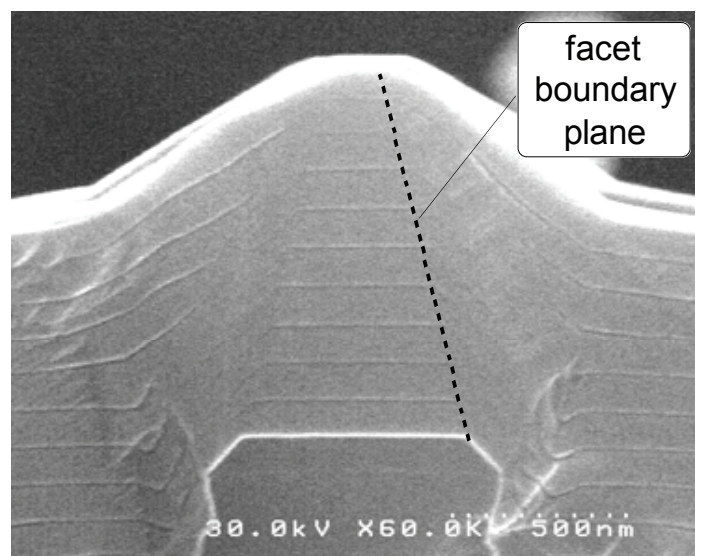

Fig.3 Cross-sectional SEM image of a $<510>$ oriented wire after repeated growth.

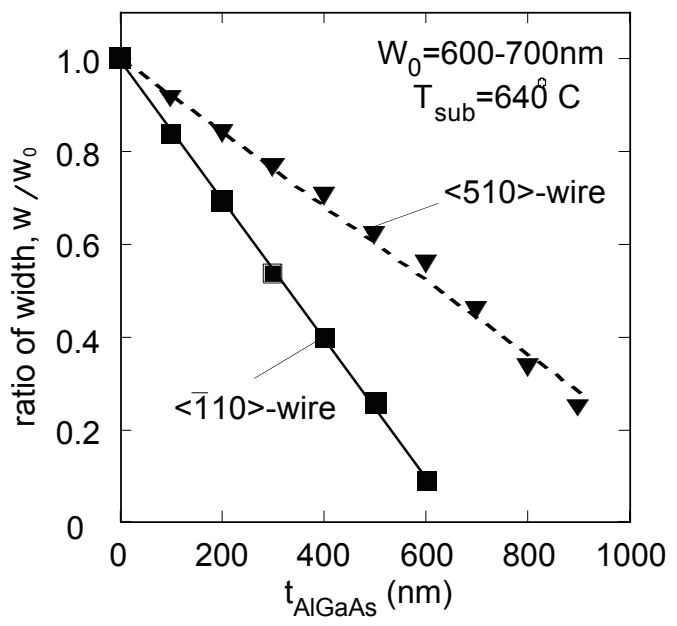

Fig.4 Normalized wire width plotted as a function of the supply thickness of AlGaAs layer 
rate between on the top and the side facets.

Thus, it has been concluded that the observed linear reduction of wire width is the result of difference in the growth rate between top facets and side facets both for $<\overline{1} 10>$ - and $<510>$-oriented ridge nano-wires. The reason for the difference in the slope between $\langle\overline{1} 10\rangle$ and $<510>$-directions in Fig. 4 is because the wire width is geometrically determined by two boundary planes whose angle with respect to the initial (001) mesa top depend on the growth facets and growth temperature.

Figure 5 plots the growth rate of the ridge top of the bottom AlGaAs layer as a function of the initial lateral ridge width, $\mathrm{w}_{0}$, for $\langle\overline{1} 10\rangle_{-}$and $<510>$ orientations. Here, the growth rate on the ridge top is normalized by that on the planar (001) substrate in such a

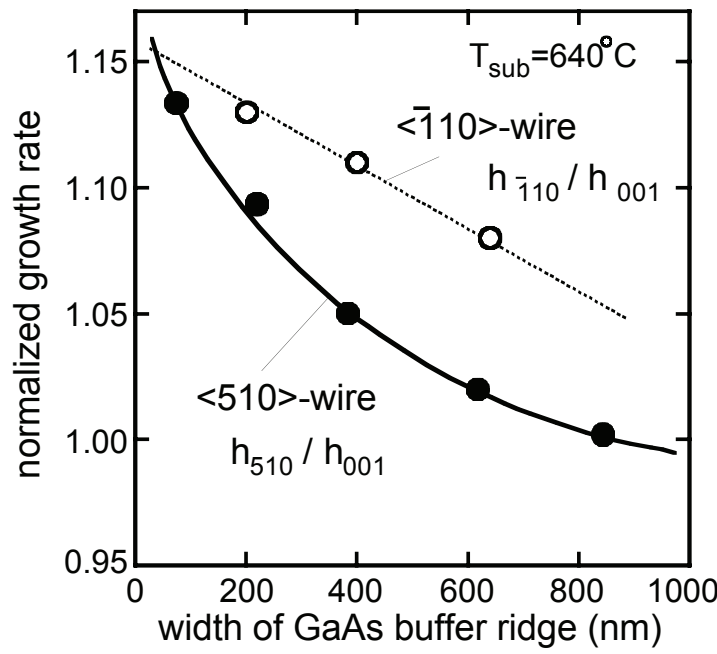

Fig.5 Normalized growth rate vs. initial lateral width

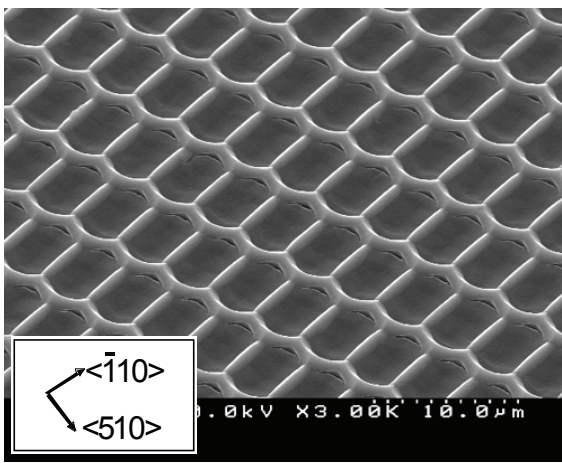

(a)

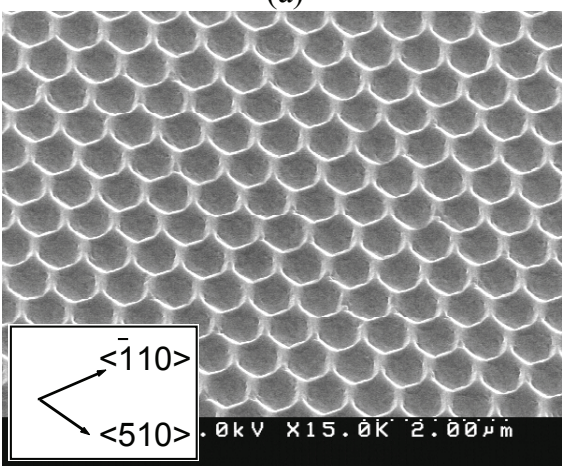

(b)

Fig.6 SEM images of MBE grown hexagonal nano-wire networks with a density of (a) $2.0 \times 10^{6} \mathrm{~cm}^{-2}$ hexagonal networks and (b) $2.4 \times 10^{8} \mathrm{~cm}^{-2}$. way that it is defined as $h_{\overline{1} 10} / h_{001}$ and $h_{510} / h_{001}$ where $h_{\overline{1} 10}$ and $\mathrm{h}_{510}$ are ridge heights shown in Fig.2 (a) and (b), respectively, and $\mathrm{h}_{001}$ is the grown thickness on the planar reference (001) substrate.

The height of nano-wires formed in both direction increased as the ridge width decreased. This is because the growth area of the ridge top facets decreases with respect to the same amount of adatom supply from both side facets. Furthermore, the results indicate that the vertical position of nano-wires can be matched for two different directions by selecting the pattern sizes.

\section{Fabrication and Characterization of Hexagonal Nano-wire Networks}

Attempts to grow hexagonal nano-wire networks were made by using the hexagonal patterns shown in Fig.1 (c). Figures 6 (a) and (b) show the top views of fabricated nano-wire networks with a node density of $2.0 \times 10^{6} \mathrm{~cm}^{-2}$ and $2.4 \times 10^{8} \mathrm{~cm}^{-2}$, respectively. The latter had a submicron pitch for hexagon. The SEM images indicate that selective growth took place by following the initial pattern with remarkable uniform morphology over a large area.

Figures 7 (a) and (b) show the results of CL and PL measurements on the hexagonal nano-wire network with the density of $2.0 \times 10^{6} \mathrm{~cm}^{-2}$. From the panchromatic CL image shown in Fig.7 (a), CL emissions from nano-wires were clearly observed on the both of $\langle\overline{1} 10\rangle$ - and $<510>$ - QWRs, and their intensities were found to be uniform over a large area.

PL measurements also showed formation of QWRs in

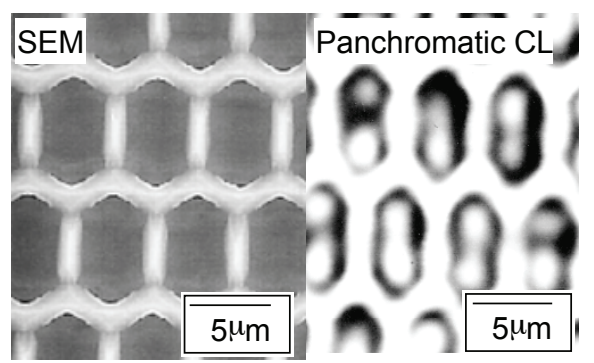

(a)

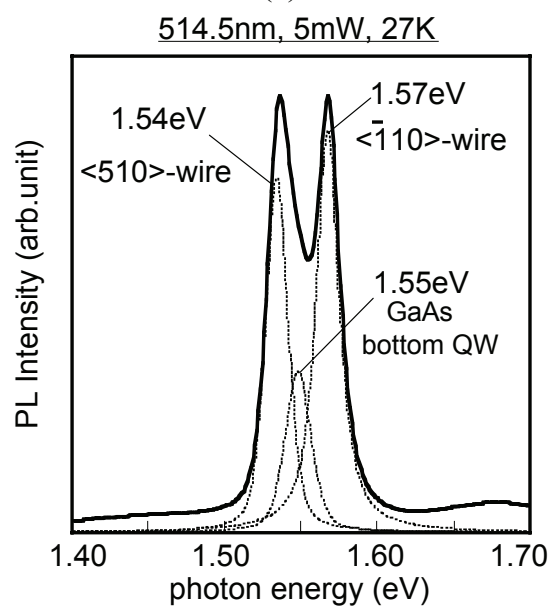

(b)

Fig.7 (a) SEM image and panchromatic CL image of the hexagonal nano-wire network. and (h) PI, snectra. 
the hexagonal nano-wire network, as shown in Fig.7 (b). Two sharp PL emissions were observed at $1.54 \mathrm{eV}$ and $1.57 \mathrm{eV}$. From a detailed spatially resolved monochromatic CL measurements, it was found that the emission peaks at $1.54 \mathrm{eV}$ and at $1.57 \mathrm{eV}$ come, respectively, from $<510>$-oriented wires and $<\overline{1} 10>$ oriented wires. PL peak positions obtained experimentally showed excellent agreements with those calculated on arrow-head shaped wire structures, using the finite difference method.

Figure 8 shows the results of AFM observation on a sample with a higher node density of $3 \times 10^{8} \mathrm{~cm}^{-2}$ after entire growth of $\mathrm{AlGaAs} / \mathrm{GaAs} / \mathrm{AlGaAs}$ layers on patterned substrates. The pattern sizes of $\langle\overline{1} 10>$ - and $<510>$-direction were designed to achieve smooth wire connections in both directions, using the results shown in Fig.4 and Fig.5. It was also found that better overall height uniformity could be obtained by making the $<\overline{1} 10>$-wire segment shorter than the $<510>$-wire segment. Line profiles shown in Fig.8 (b) were taken along $\langle\overline{1} 10>$ - and $<510>$-directions, including the points, A, B and C shown on the AFM image in Fig.8 (a). The AFM line profiles indicate that the top surface of the grown sample maintains almost the same height both in the $\langle\overline{1} 10>$ - and $<510>$-directions, although it becomes slightly lower by about $5 \%$ at the middle of the $<510>$-wire segment. The latter may be due to the small change of the atom incorporation rate between the crossing points and the center of the $<510>$-wire segment. The surface morphology was also excellent with extremely small rms roughness values below $1.0 \mathrm{~nm}$.

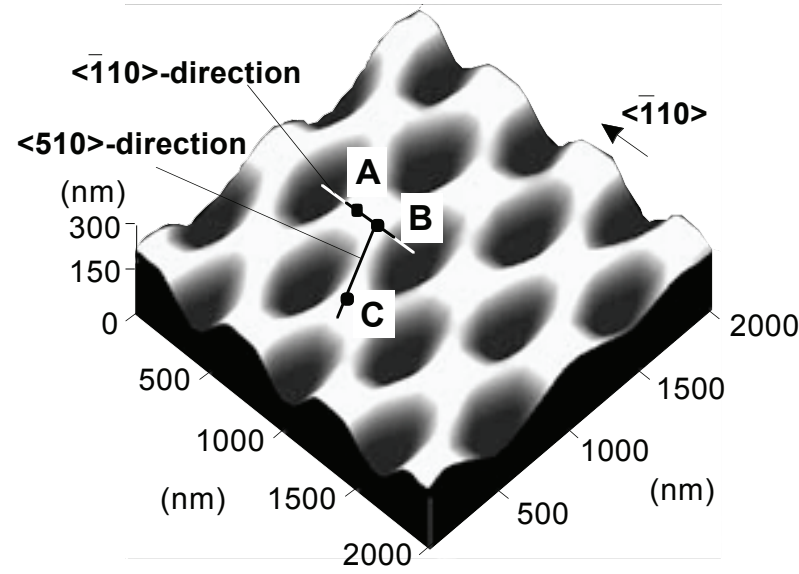

(a)
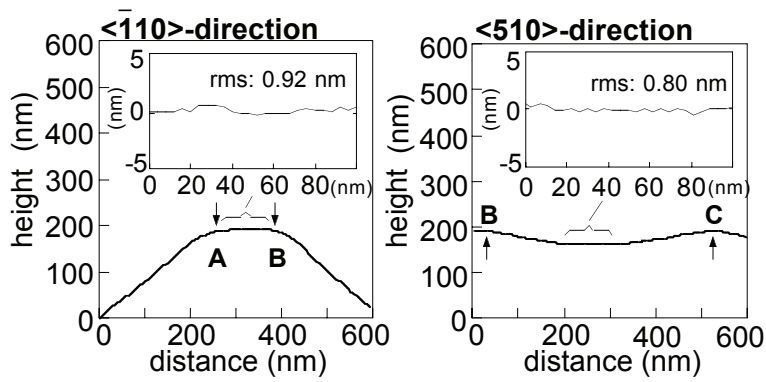

(b)

Fig.8 (a) AFM image of the hexagonal nano-wire network with a node density of $3 \times 10^{8} \mathrm{~cm}^{-2}$, and (b) its line profile along the $<\overline{1} 10>$ - and $<510>$-direction.
Thus, from the AFM profiles combined with the uniformity of CL emission shown in Fig.7 (a), it seems highly likely that smooth connection has been achieved between $<\overline{1} 10>$ - and $<510>$-oriented inner wire segments. However, future direct electrical measurements are required to directly confirm inner wire connections.

\section{Conclusion}

In view of applications to hexagonal BDD quantum circuits, growth of hexagonal GaAs nano-wire networks was attempted by selective MBE growth on (001) pre-patterned substrates.

(1) The wire width of $<\overline{1} 10>$ - and $<510>$ - GaAs QWR is determined by growth boundary planes resulting from the difference in the migration rate and the atom incorporation rate between top facets and side facets of the GaAs ridge structure. Thus, the wire width can be kinetically controlled precisely by growth conditions.

(2) From SEM, PL and CL measurements, uniform and smoothly connected hexagonal nano-wire networks were fabricated on the pattern formed combining of $\langle\overline{1} 10>$ and $<510>$-wire directions. These results indicate that the present growth technique is very promising to realize a high density nano-wire network for BDD-based quantum LSIs.

\section{Acknowledgement}

The work reported here is supported in part by $21 \mathrm{st}$ Century COE Project on "Meme-Media Technology Approach to the R\&D of Next-Generation Information Technologies" from Japanese Government.

\section{References}

[1] E. Kapon, D. M. Hwang and R. Bhat: Phys. Rev. Lett., 63 (1989) 430.

[2] T. Fukui, S. Ando, Y. Tokura and T. Toriyama: Appl. Phys. Lett., 58 (1991) 2018.

[3] S. Koshiba, H. Noge, H. Akiyama, T. Inoshita, Y. Nakamura, A. Shimizu, Y. Nagamune, M. Tsuchiya, H. Kano, H. Sakaki and K. Wada: Appl. Phys. Lett., 64 (1994) 363.

[4] H. Fujikura and H. Hasegawa: J. Electron. Mater., 25 (1996) 619.

[5] X. L. Wang, M. Ogura and H. Matsuhata: Appl. Phys. Lett., 66 (1995) 1506.

[6] T. Sato, I. Tamai C. Jiang and H. Hasegawa: Inst. Phys. Conf. Ser., 170-4 (2002) 325.

[7] T. Sato. I. Tamai and H. Hasegawa: presented at ISCS 2002.

[8] H. Hasegawa and S. Kasai: Physica E, 11 (2001) 371. [9] S. Kasai and H. Hasegawa: IEEE Electron Device Letters, 23 (2002) 446. 